\title{
Mission Concept: Cave and Lave Tube Exploration on Moon, Mars and Icy Moons for Eventual Settlement
}

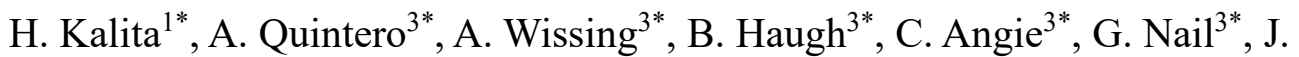

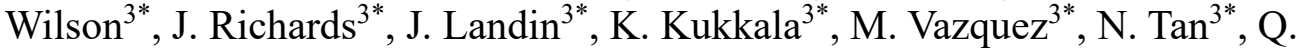 \\ Lamey $^{3^{*}}$, R. Lu ${ }^{3 *}$, R. Peralta, V. Vilvanathan ${ }^{3 *}$, R. McCandless ${ }^{4} \mathrm{~J}_{\text {. Thangavelautham }}{ }^{2}$ \\ ${ }^{1}$ Space and Terrestrial Robotic Exploration (SpaceTREx) Laboratory, Aerospace and \\ Mechanical Engineering Department, University of Arizona, 1130 N Mountain Ave, \\ Tucson, AZ 85721; PH (480) 274-3146; email: hkalita@email.arizona.edu \\ ${ }^{2}$ Space and Terrestrial Robotic Exploration (SpaceTREx) Laboratory, Aerospace and \\ Mechanical Engineering Department, University of Arizona, 1130 N Mountain Ave, \\ Tucson, AZ 85721; PH (617) 800-7497; email: jekan@email.arizona.edu \\ ${ }^{3}$ Asteroid Science, Technology and Exploration Research Organized by Inclusive \\ eDucation (ASTEROID) Laboratory, Aerospace and Mechanical Engineering \\ Department, University of Arizona, 1130 N Mountain Ave, Tucson, AZ 85721. \\ 4 SignalLuna, Ireland/US
}

\section{SHORT ABSTRACT}

Future missions will be targeting extreme and rugged environments such as pits, caves, canyons, cliffs and crater rims of the Moon, Mars and icy moons. We propose multiple small, robots called SphereX that would explore these sites with the intent on setting human bases that would be naturally shielded from surface hazards.

\section{REGULAR ABSTRACT}

The next frontier in solar system exploration will be missions targeting extreme and rugged environments such as pits, caves, canyons, cliffs and crater rims of the Moon, Mars and icy moons. These environments are time capsules into early formation of the solar system and will provide vital clues of how our early solar system gave way to the current planets and moons. These sites will also provide vital clues to the past and present habitability of these environments. Current landers and rovers are unable to access these areas of high interest due to limitations in precision landing techniques, need for large and sophisticated science instruments and a mission assurance and operations culture where risks are minimized at all costs. This research presents an overview of mission concepts using small, low-cost robots and landers that can be deployed to access these extreme environments. A credible solution is to develop an architecture that permits taking high exploratory risks that translates into high reward science but without compromising the rest of the mission. Rapid advancement in electronics, sensors, actuators and power supplies have resulted in ever-shrinking devices and instruments that can be housed in small platforms. We propose to use multiple small, low-cost, modular spherical robots called SphereX that are several kilograms in mass and several liters in volume and is designed to hop and roll short distances. A large rover or lander may carry several of these SphereX robots that are tactically deployed to explore and access rugged environments inaccessible by it. The 
landers and rovers to carry these SphereX robots can also be designed in the CubeSat form factor using readily available Commercially-Off-The-Shelf (COTS) components. Moreover, once these extreme environments are explored, the planetary pits which are theorized to be remnant lava tubes offers ideal conditions for setting up a human base. The pits offer natural shelter from existing dangerous surface conditions and hazards such as temperature fluctuations, radiation, and meteorite impacts.

\section{INTRODUCTION}

In the next few decades, we aspire to send human and robotic explorers to every corner of our solar system to perform orbital, surface and even subsurface exploration. These explorers will pave the way towards identifying the diverse surface environments, physical processes and structure of the planets and small bodies answering fundamental questions about the origins of the solar system, conditions to sustain life and prospects for resource utilization and off-world human settlement. Achieving this major exploration milestone remains technologically daunting but not impossible. An emerging target are the extreme environments of the Moon, Mars and icy moons, including pits, caves, canyons, cliffs, skylights and craters as shown in Fig. 1. These are high-priority targets as outlined in the Planetary Science Decadal survey [1]. These environments are rich targets of origin studies, as the rocks and surfaces carved out from natural processes expose a time-record of ancient events including changing climate and surface composition, violent impacts/collisions events and evidence for organic chemicals. Moreover, caves offer natural shelter from radiation, harsh surface processes such as dust storms and are generally insulated by the varying high and low external temperatures. These conditions could harbor isolated, ancient ecosystems.
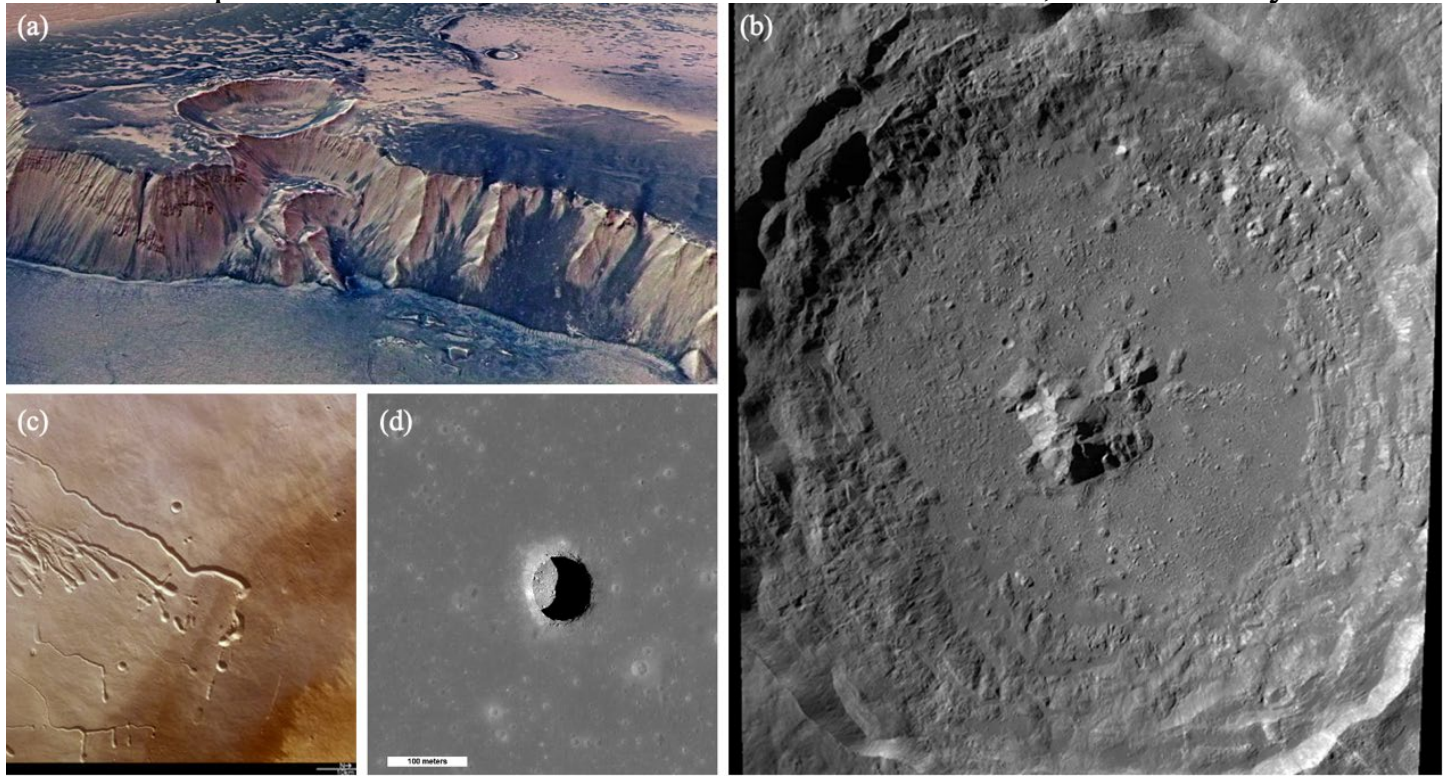

Fig. 1. Extreme environments of the Moon and Mars: (a) High cliffs surrounding Echus Chasma on Mars (nasa.gov), (b) Tycho crater on Moon (NASA/Goddard/Arizona State University), (c) Lava tubes on Pavonis Mons on Mars (ESA), and (d) Mare Tranquilitatis pit on Moon (NASA/GSFC/Arizona State University). 
High resolution orbital imagery from the Lunar Reconnaissance Orbiter Camera (LROC) reveal evidence for subsurface voids and mare-pits on the lunar surface $[2,3]$. Similar discoveries have been made with the HiRise camera onboard the Mars Reconnaissance Orbiter (MRO) observing the Martian surface. These accessible voids could be used for a future human base because they offer a natural radiation and micrometeorite shield and offer constant habitable temperatures of -20 to $-30^{\circ} \mathrm{C}$ [4]. Exploration of these extreme and rugged environments remains out of reach from current planetary rovers and landers; however, the 2015 NASA Technology Roadmaps prioritizes the need for next-generation robotic and autonomous systems that can explore these extreme and rugged environment [5]. The challenges are three-fold and stem from current landing technology that requires wide-open spaces with no obstacles or landing hazards. A second challenge stems from current planetary vehicle architectures. Planetary rovers and landers ever since Luna 9, the first mission to softland on the Moon, have been generally growing in size and capability to house a growing variety of sophisticated science instruments. A third challenge has been the high standards of mission assurance expected. Due to the high costs and prestige for the nations involved, any form of exploratory risk that may reduce the life of the mission or result in damage to one or more subsystems is avoided. This is despite the potential science rewards from taking these exploratory risks.

A credible solution is to develop an architecture that permits taking high exploratory risks that translates into high reward science but without compromising the rest of the mission. Rapid advancement in electronics, sensors, actuators and power supplies have resulted in ever-shrinking devices and instruments that can be housed in small platforms. This has resulted in the wide adoption of CubeSats for Low Earth Orbit (LEO) missions and technology demonstrations. CubeSats are emerging as platform for performing high-risk, high-reward interplanetary exploration [6]. The technology uses Commercial-Off-The-Shelf (COTS) technology with adaptations to the space environment. Further technological advancement is leading towards radiation hardened version of these components for use in deep-space and planetary environments. However, these technologies still need to be proven in these planetary environments.

Moreover, the proposed Lunar Gateway (Fig. 2) will play an important role as a forward refueling base/pit stop on a journey to the Moon. Current plans are to position the base well outside the gravity wells of Earth and the Moon. A forward base such as the Lunar Gateway is needed to serve as a site for repairs/logistics hub and refueling depot in between any long journey to the Moon

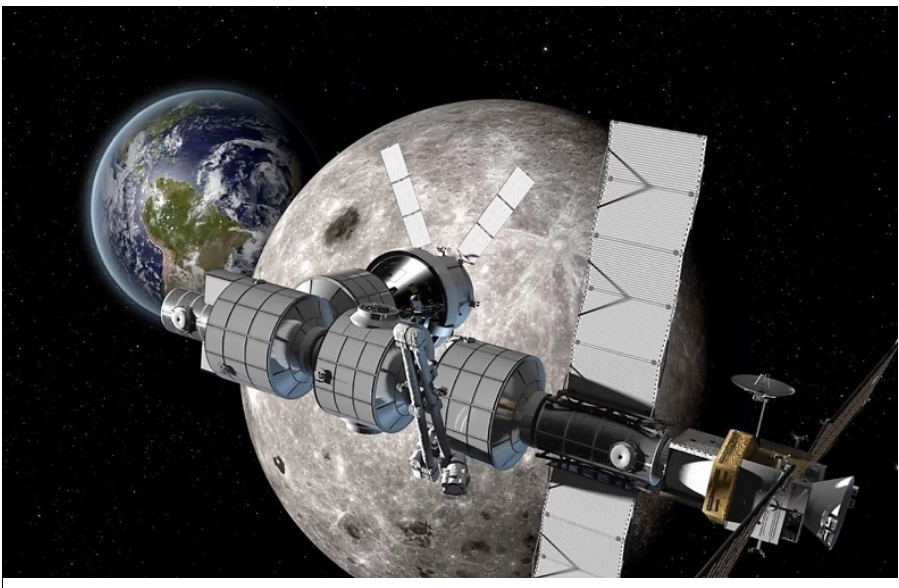

Fig. 2. Current concepts of the Lunar Gateway (courtesy of NASA). 
and beyond. This contrasts with Zubrin's Moon Direct approach [7]. While it is true the Apollo Program didn't require a hub or a forward base, it made the program all the more expensive and daring, that it couldn't be repeated for over $50+$ years. A better solution is needed that provides a steppingstone to the Moon as part of a long-term, sustainable, two-prong strategy of science exploration and human colonization. Long term human colonization of the Moon requires further exploration of the lunar surface to better understand the origin, the formation and its composition in ever more detail. Significant insight has been obtained from the orbiting Lunar Reconnaissance Orbiter (LRO); however complementary surface missions are required to further our insight. Current plans for lunar science mission are all relatively expensive as they need to account for the cost of launch, upper-stage and the spacecraft. Use of the Lunar Gateway as a launch-pad for lunar flyby, orbit and surface mission can significantly cut down costs. The miniaturization of spacecraft electronics, sensors and actuators and recent demonstration of the MarCO Mars CubeSats [8] show that CubeSats despite being at their infancy as interplanetary explorers could be the ideal platform/vehicles for these flyby, orbit and surface missions to the Moon [9].

Compared to the SLS EM1 mission, the Lunar Gateway can play an important role not just a drop-off point but also as a permanent communication relay/hub to the CubeSats operating on the Moon. It may even be worthwhile to control and monitor the CubeSats from the Gateway rather Earth to ease communication congestion of the DSN. The relatively short distances could enable teleoperation of these CubeSats as opposed to fully autonomous operations. This could further simplify operation and minimize risks/uncertainties. The Lunar Gateway could also be used to insert an orbiting lunar relay and global positioning system to further facilitate exploration of both the far-side and near side of the Moon. Importantly these assets can increase localization accuracy to $10 \mathrm{~s}$ of $\mathrm{cm}$ position accuracy and communication from with far-side assets. The relay and GPS system could be composed of CubeSats and such mission will further advance miniaturized ADCS systems for use in deep space.

Just as sending CubeSats to Low Earth Orbit (LEO) has become routine, it will be possible to do the same to the lunar vicinity. Sending these CubeSats to perform critical science exploration avoids the costs and risks of sending humans. The end to end mission costs could be reduced to $\$ 20$ million and below for $54 \mathrm{~kg}, 27 \mathrm{U}$ CubeSats. Secondarily, the CubeSats being disposal can be used to further advance low-cost technology to navigate and precision land on the lunar surface, while advancing critical technologies like propulsion and communication which remain important technological hurdles $[6,10,11]$. The platform and the location maybe the ideal proving ground to test next-generation hybrid propulsion technologies that enables the CubeSats to perform sample-return from the lunar surface. Once lunar surface missions become routine, it may be possible to tackle Planetary Science Decadal questions including assembling telescopes and sensor networks on the far-side of the Moon [1].

Motivated by these ideas, we present an architecture of small, low-cost, modular spherical robot called SphereX that is designed for exploring extreme environments in low-gravity planetary bodies like the Moon, Mars, icy moons and asteroids through 
hopping and rolling [12-15]. It consists of space grade electronics like computer board for command and data handling, power board for power management and radio transceiver with multiple $\mathrm{S} / \mathrm{X}$-band antennas for communicating among multiple robots. Mobility is achieved through the combined action of a propulsion system consisting of one thruster along with an attitude control system consisting of 3 reaction wheels to perform controlled ballistic hopping. The 3-axis reaction wheel system can also be used for controlled rolling mobility. Moreover, it consists of a power system for power generation, storage and distribution among all the subsystems and a thermal system comprising of several active and passive elements to maintain its desired body temperature for survival. The rest of the volume is accompanied by payloads in the form of a pair of stereo cameras for imaging and a LiDAR system for mapping and navigation. A large rover or lander may carry several of these SphereX robots that can be tactically deployed to explore and access extreme environments inaccessible by it as shown in Fig. 3.

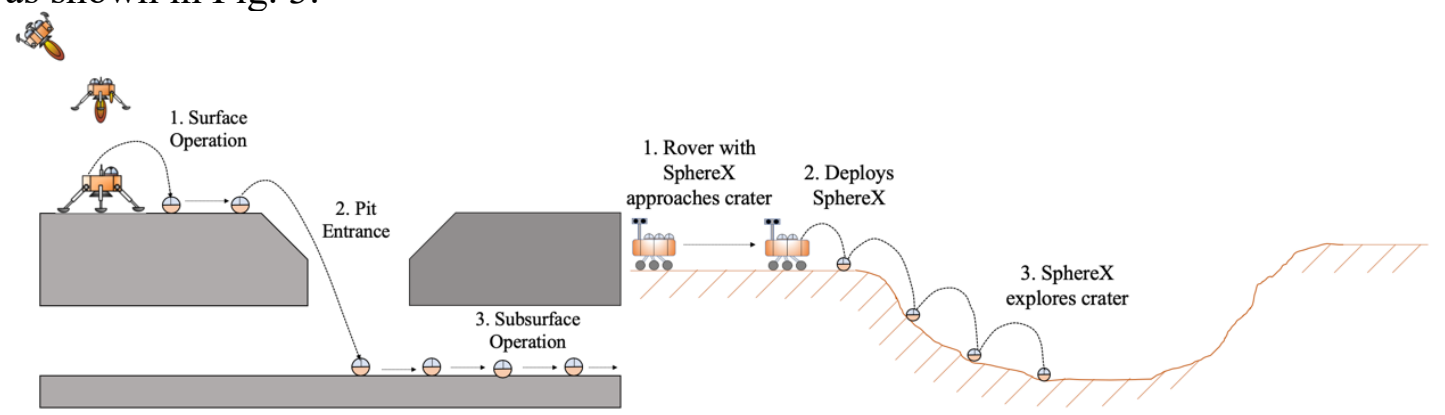

Fig. 3. (Left) Concepts of operation for exploring Lunar pits, (Right) Concepts of operation for exploring planetary craters.

\section{MISSION CONCEPT FOR EXPLORING LUNAR PITS}

Recently discovered lunar mare "pits" are key science and exploration targets. The first three pits were discovered within Selene observations and were proposed to represent collapses into extant lava tubes [16]. Subsequent Lunar Reconnaissance Orbiter Camera (LROC) images revealed 5 new mare pits and showed that the Mare Tranquilitatis pit (MTP; $8.335^{\circ} \mathrm{N}, 33.222^{\circ} \mathrm{E}$ ) opens into a sublunarean void at least 20 meters in extent (Fig. 2) [2,3]. The pit diameters range from 86 to $100 \mathrm{~m}$ with a maximum depth from shadow measures of $\sim 107 \mathrm{~m}$. Several large, angular blocks are sparsely distributed across the floor, and likely represent detritus from the pit walls or collapsed roof materials. Additionally, more than 200 pits were discovered in impact melt deposits. Current theories suggest there are vast networks of lava-tubes stretching $100 \mathrm{~s}$ of kms. The suspected lunar lava tubes are expected to be much larger in size than those found on Earth, with a diameter of 80 to $100 \mathrm{~m}$. Several of these lunar pits are found near the poles and conditions are sufficient to harbor water-ice. Entering and exploring these lunar pits are of high priority. The lunar pits being sheltered from the surface could hold records of the lunar geo-history without being impacted by cosmic radiation, surface weathering and from micro-meteorites impacts. Furthermore, the temperature inside the pits are expected to be around $-25^{\circ} \mathrm{C}$. These factors make the pits idea locations to locate a future human base or park valuable lunar assets. 
Landing a CubeSat in the vicinity of a lunar pit presents some important GNC challenge. The main challenge includes precision landing near or inside the pit. This will require visual navigation techniques. One possibility includes looking for shadow lines inside the pit and lock on the target. Landing inside the pit presents some exciting outcomes but can serve to limit the length of the mission. Other options include landing nearby the pit and deploying smaller SphereX (Pit Bots) into the pit. One of the key tasks concerning pits is determining their subsurface extents, and thus fully understanding their exploration and scientific value. Key measurement objectives include determination of the extent of sublunarean void to a range of at least 100 meters, understanding its composition and detecting the presence of water in the regolith, measurement of the thermal environment on the MTP floor and within the void and mapping of the void. Here, we present an example of lunar CubeSat that could be deployed from the Lunar gateway to perform exciting surface science. The mission concept called Arne-II $[17,18]$ will be used to explore the lunar pits such as Mare Tranquilitatis. The lander is a $27 \mathrm{U}$ and has mass of $54 \mathrm{~kg}$ and it is expected to last no longer than 12 days. This avoids the thermal challenges of surviving the lunar night and simplifies the spacecraft design. The lander will be self-propelled utilizing greenmonoprop rockets with a maximum delta-v of $2.5 \mathrm{~km} / \mathrm{s}$. Arne II requires precision landing and will be required to land within 500 meters of Mare Tranquilitatis. Through this mission, significant insight will be learned of lunar geology and geo-history and will provide insight into developing a future human base. 


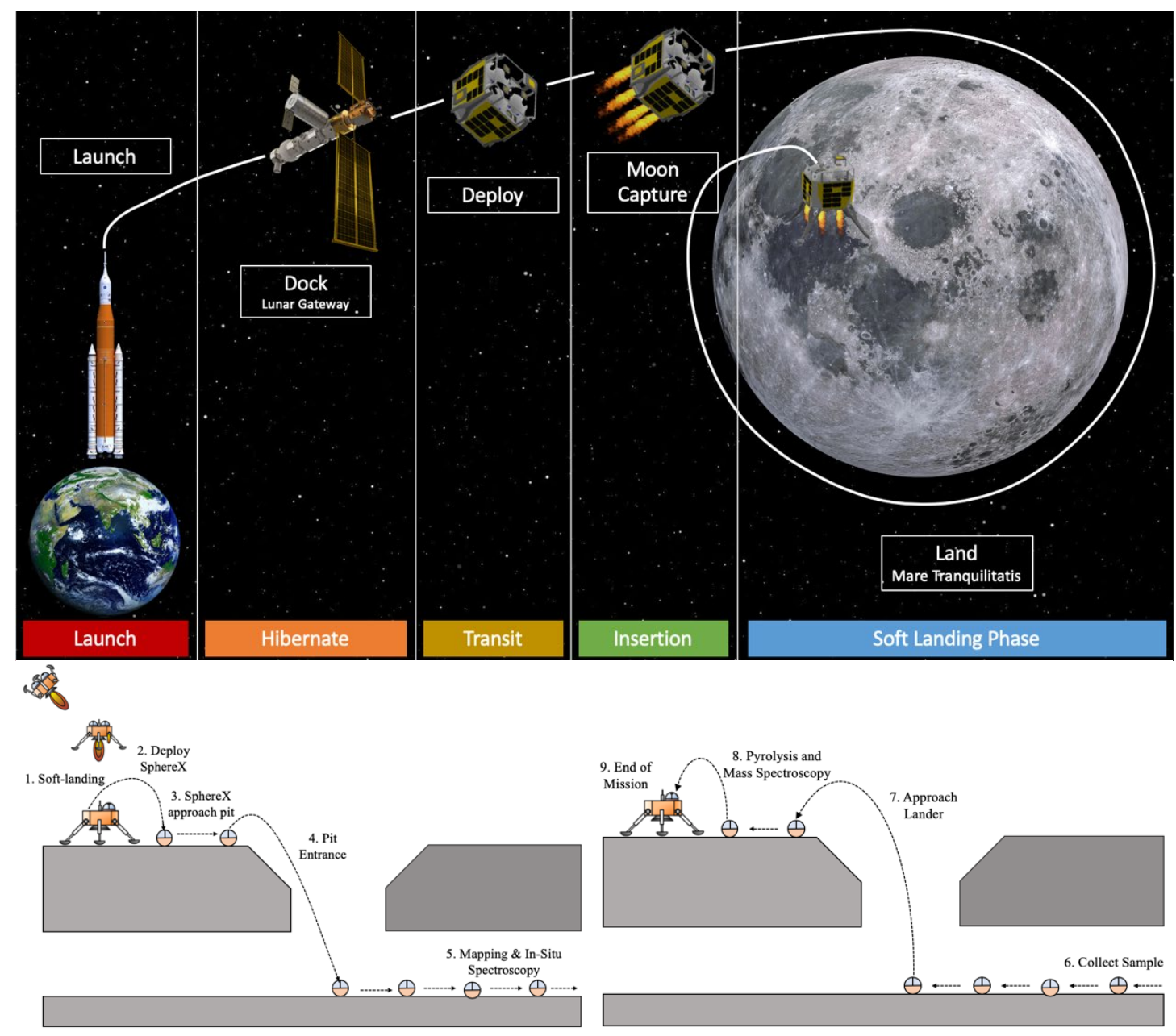

Fig. 4. (Top) Concept of Operations of Arne-II from Earth launch to Moon landing, (Bottom) Concept of operations of SphereX after Moon landing.

Figure 4(Top) shows the first phase of the mission. The CubeSat will be launched on NASA's deep space rocket, the Space Launch System (SLS) where it will be encapsulated, docking with the Lunar Gateway presumed to be located at Lagrangian L2 point. The lander will be stored on the outside of the Gateway logistics module. The lander will be in a hibernated state until its ready for science deployment. When ready the CubeSat will be loaded into a P-Pod and launched on its way to the Lunar insertion orbit. The lander has its own propulsion, attitude-determination and control system and communication system. The lander has a total delta-v of $2.5 \mathrm{~km} / \mathrm{s}$ using its High-Performance Green Propulsion (HPGP) system. After the lander performs its lunar orbit insertion maneuver, it will perform another impulsive burn to target the landing site. On its way to the landing site, at about $25 \mathrm{~km}$ from the surface it will prepare to land. The onboard camera and lens system will be used to navigate and the propulsion system to perform soft landing on the landing site. The CubeSat lander upon successfully landing will deploy the three SphereX robots using a spring deployment system. The SphereX robots will then hop near the pit entrance, enter the pit and start mapping using the onboard 3D LiDAR sensors and stereo cameras as shown in Fig. 4(Bottom). Each robot will also take electrical impedance spectroscopy measurements to determine the water content, distribution, and phase in the planetary regolith inside. 
Each robot will also collect a few grams of regolith sample and then hop back to the lander. The collected samples will then be inserted inside the VAPoR instrument to perform pyrolysis and mass spectrometry. The CubeSat lander will then transmit the data (electrical impedance spectroscopy, pyrolysis and mass spectroscopy, 3D point cloud data of the pit, images and videos of the landing process, and images and videos of the robots hopping inside the pit) back to the Lunar Gateway. Estimated mission length is 3 days. The lander will land during daytime and there are no plans for the lander to survive the lunar night which simplifies the mission.

\section{SPACECRAT DESIGN}

The proposed lander is a $27 \mathrm{U}(34 \times 35 \times 36 \mathrm{~cm})$ CubeSat (Fig. 5 and 6). The lander will be deployed from the Gateway to the Lunar surface. The lander is equipped with four 22N HPGP and four $100 \mathrm{mN}$ HPGP thrusters. The High-Performance Green Propulsion (HPGP) provides higher specific impulse and higher propellant density, which results in increased performance compared to traditional propulsion. The propellant is based on AND (Ammonium DiNitrimide) and is considered less toxic, non-carcinogenic and simpler to handle than hydrazine. The onboard Attitude Determination Control System (ADCS) consists of the Blue Canyon Technologies XACT-50. Reaction wheel desaturation will be performed using the thrusters. The power system consists of GOMspace NanoPower BPX rechargeable lithium ion batteries, MMA eHaWK solar PV and the power electronics from GOMspace NanoPower p60 system. The lander will also use $3 \mathrm{~S}$-band antennas and a S-band transceiver. The C\&DH will be performed by Space Micro CSP (CubeSat Space Processor) which is a compact single board computer designed around Xilinx Zync7020. The chassis and the landing legs will be custom built. The instruments in the lander includes a pyrolytic analyzer (pyrolysis oven coupled to a mass spectrometer) for vacuum pyrolysis of regolith to identify the presence of water, and a camera and a lens system integrated with Xilinx Vertex-5QV FPGA for navigation during landing phase. The lander will also carry three spherical hopping robots (SphereX) which will be deployed near the entrance of a lava tube. The SphereX robots will hop inside the lava tube for mapping, detecting the presence of water and collecting samples. 


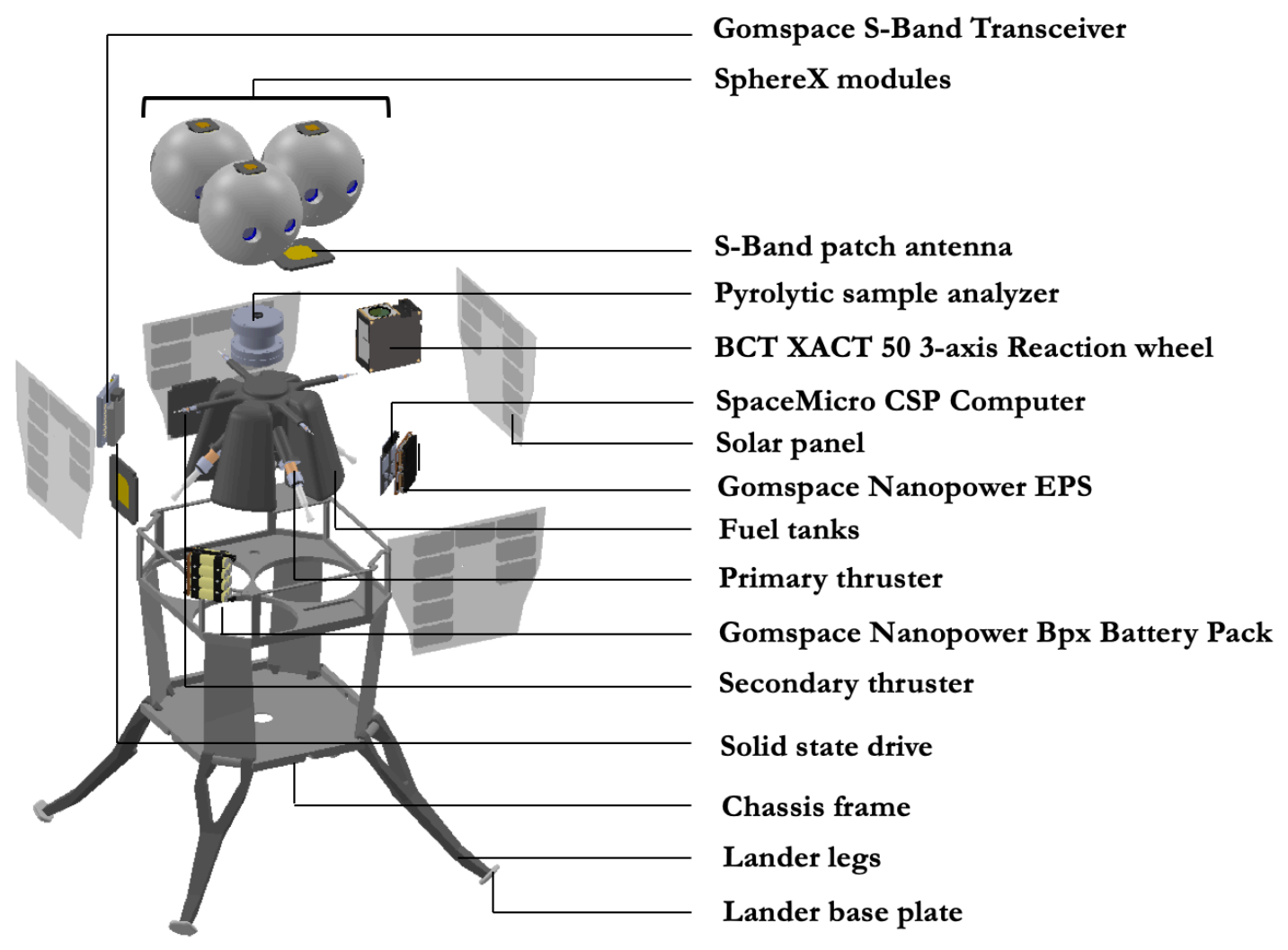

Fig. 5. Detailed description of the components of the CubeSat Lander (Arne-II).
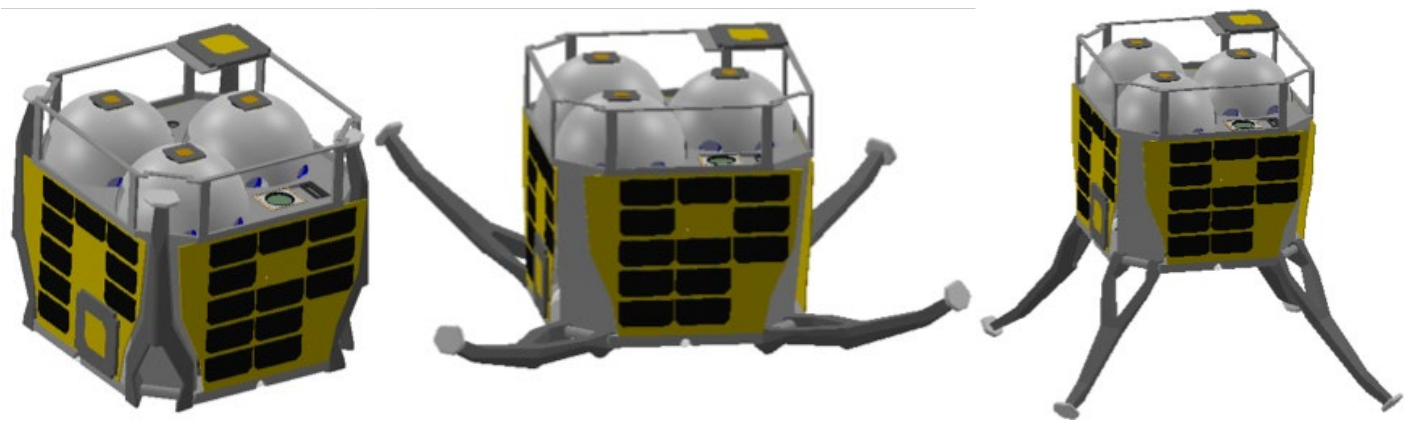

Fig. 6. Sequence of deployment of the landing legs.

\section{Propulsion System}

Compared to traditional propulsion, High Performance Green Propulsion (HPGP) provides higher specific impulse and higher propellant density, which results in increased performance. The propellant is based on AND (Ammonium DiNitrimide) and is considered less toxic, noncarcinogenic and simpler to handle than hydrazine. The architecture of HPGP propulsion systems consists of COTS (Commercial-Off-The-Shelf) components with extensive flight heritage. This enables a simplified transition away from hydrazine and allows
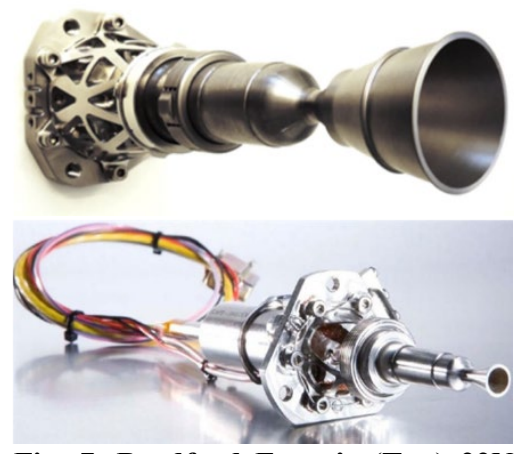

Fig. 7. Bradford Ecaps's (Top) 22N HPGP thruster (Bottom) $100 \mathrm{mN}$ the overall mission cost to be reduced. The propulsion system consists of four $22 \mathrm{~N}$ 
HPGP, four 100mN HPGP thrusters and a propellant tank (Fig. 7). The $22 \mathrm{~N}$ thrusters will be used to perform delta-v maneuvers and soft landing, while the $100 \mathrm{mN}$ thrusters will be used for positional correction along the horizontal plane during landing. Table 1 provides the details of the thrusters selected for Arne-II. Since, the lander will be deployed from the Earth-Moon L2 Lagrange point, it will require a delta-v of $2.5 \mathrm{~km} / \mathrm{s}$ to successfully perform soft landing on the surface of the Moon. Considering the average specific impulse of the $22 \mathrm{~N}$ HPGP Thruster to be $245 \mathrm{~s}$, the amount of propellant required is calculated according to the Tsiolkovsky rocket equation:

$$
\Delta v=I_{s p} g_{0} \ln \frac{m_{0}}{m_{0}-m_{p}}
$$

Where, $\Delta v$ is the required delta-v, $I_{s p}$ is the specific impulse of the propellant, $g_{0}$ is the standard gravity, $m_{0}$ is the initial total mass of the lander, and $m_{p}$ is the mass of the propellant.

Table 1. Details of the thrusters used for Arne-II.

\begin{tabular}{lcc}
\hline \hline \multicolumn{1}{c}{ Parameters } & 22 N HPGP Thruster & 100 mN HPGP Thruster \\
\hline Thrust Range & $5.5-22 \mathrm{~N}$ & $30-100 \mathrm{mN}$ \\
Nozzle Expansion Ratio & $150: 1$ & $100: 1$ \\
Inlet Pressure Range & $5.5-24 \mathrm{Bar}$ & $2.3-4.5 \mathrm{Bar}$ \\
Specific Impulse & $243-255 \mathrm{~s}$ & $196-209 \mathrm{~s}$ \\
Length & $260 \mathrm{~mm}$ & $55 \mathrm{~mm}$ \\
Mass & $1.1 \mathrm{~kg}$ & $0.040 \mathrm{~kg}$ \\
Maturation Level & TRL 5/6 & TRL 5/6 \\
\hline \hline
\end{tabular}

Navigation System

A camera and a lens system will be used for navigating the lander to its landing site. A high-resolution wide-angle camera system (Fig. 8) is selected to allow for a single landing navigation camera to minimize subsystem mass and volume. 0.55 meter per pixel imagery of the landing site is available from the Lunar Reconnaissance Orbiter Camera (LROC). Our selected camera system provides 595 meter per pixel resolution at an altitude of $25 \mathrm{~km}$, with a total coverage of over 21,000 square kilometers of lunar surface at that same altitude. At $1 \mathrm{~km}$ altitude the camera resolution will be 0.95 meter per pixel. Assuming a uniform distribution of craters, we should expect roughly one 5-20 kilometer crater

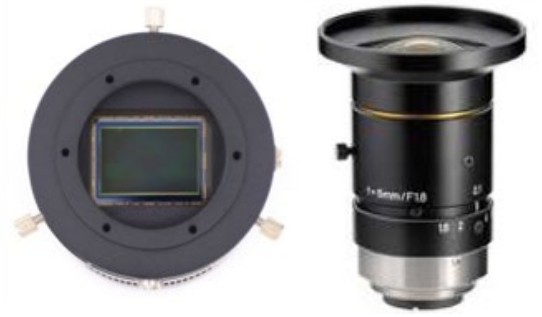

Fig. 8. (Left) QHY367C cooled full frame CMOS color camera, (Right) Edmund Optics 5mm FL ultra-high resolution fixed focal length lens. every 1000 square kilometers, providing an estimated 21 large craters to be used for visual odometry and navigation at $25 \mathrm{~km}$. During landing, the navigation camera will stream imagery to the FPGA and match with the preloaded features, providing position and velocity estimate of the craft. The position estimate is estimated to be three orders of magnitude better than current inertial estimates [19]. The expected target deviation from the landing site is expected to be roughly one meter according to studies of Mars Science Laboratory (MSL) touchdown imagery [19]. 


\section{Instruments and Payloads}

The extraction and identification of volatile resources including water, oxygen, and hydrocarbons from the regolith on the Moon can be done by vacuum pyrolysis at elevated temperatures that releases volatiles trapped inside solid samples. With a high temperature pyrolysis oven coupled to a time-of-flight mass spectrometer instrument called Volatile Analysis by Pyrolysis of Regolith (VAPoR), solid samples can be heated to high temperatures in vacuum to determine the composition of volatiles released as a function of temperature $[20,21]$. The VAPoR pyrolysis oven consists of a heated sample cup enclosed in radiation shielding (Fig. 9). The sample cup is made of conical singlestranded tungsten wire conformally

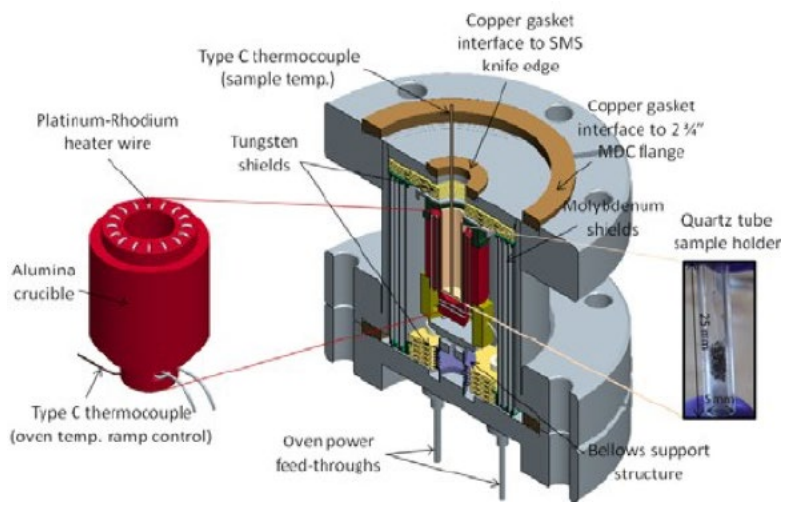

Fig. 9. Volatile Analysis by Pyrolysis of Regolith (VAPoR) instrument. coated with zirconia ceramic. In order to release oxygen from silicate minerals and some noble gas species a higher temperature in the order of $1200^{\circ} \mathrm{C}$ is required. However, water can start to be released at temperature $100^{\circ} \mathrm{C}$ to $100^{\circ} \mathrm{C}$ at $8 \mathrm{e}-6$ torr pressure [21]. The evolved gases are then inserted into a miniature MEMS time-of-flight mass spectrometer [22] to detect its major constituents. The time-of-flight mass spectrometer operates by ionizing a sample gas by electron impact, accelerating the ions to a known kinetic energy using an electric field, and allowing the ions to separate in time along a single trajectory according to their masses. For a known kinetic energy, $K E$, and flight path length, $L$, we can describe the time-of-flight, $t$, as a function of mass, $m$ :

$$
t=L \sqrt{\frac{m}{2 K E}}
$$

Heavier masses will exhibit a longer time-of-flight than lighter masses, from which a mass spectrum can be derived. The longer the flight path, $L$, the better the separation between two adjacent masses.
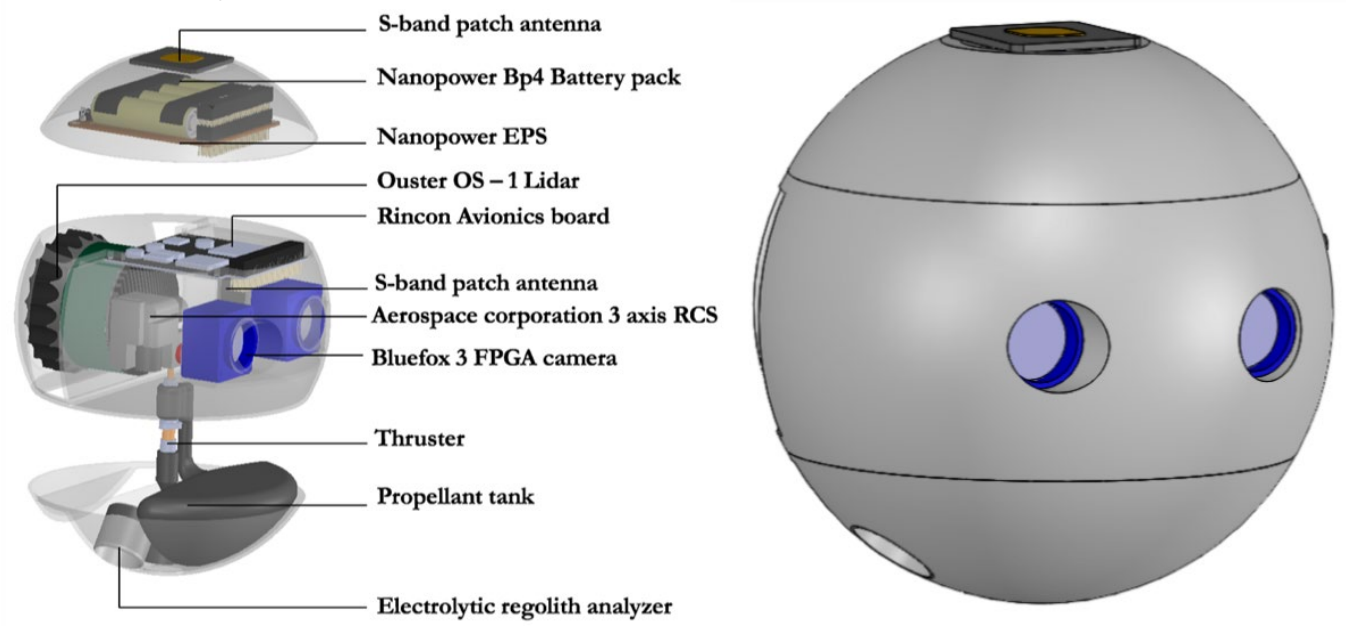

Fig. 10. Internal (Left) and External (Right) view of SphereX. 
In addition to the VAPoR instrument, Arne-II will carry three SphereX robots. SphereX is a spherical hopping robot of mass $1.5 \mathrm{~kg}$ and a diameter of $180 \mathrm{~mm}$ as shown in Fig. $10[25,26]$. The lander will deploy them near the entrance of a pit (Mare Tranquilitatis). The SphereX robots will approach the pit entrance and then hop inside the pit for exploration. The top half of the SphereX contains lithium ion batteries and EPS board for power and EnduroSat S-band patch antenna for communication. The middle section consists of the C\&DH board, 3-axis reaction wheel from Aerospace Corporation for attitude control, a pair of Bluefox 3 FPGA cameras for imaging and navigation and a 3D LiDAR scanner for mapping, navigation and localization. The bottom half consists of a $5 \mathrm{~N}$ thruster along and a propellant tank. Moreover, each of the SphereX consists of an instrument that uses impedance spectroscopy to determine water content, distribution, and phase in planetary regolith. The instrument consisting of 4 electrodes provides a detailed electrical characterization of the regolith, which offers the potential of significantly increased sensitivity to water and ice [23]. All materials have unique characteristic responses to electromagnetic stimulation that can be used to identify them. At high frequencies (infrared), the response is due to the stretching of individual bonds within the water molecule. At lower frequencies $(\mathrm{GHz})$, the orientational polarization of the water molecule itself provides a characteristic response. This polarization occurs at $\sim \mathrm{kHz}$ frequencies for water ice. Water, in particular, has a strong dipole associated with the hydrogen-bonding network. An additional interfacial polarization also occurs at water/electrode or water/regolith interfaces due to charge balance considerations at these interfaces. The frequency dispersion of the $\mathrm{AC}$ impedance spectra is caused by relaxation and resonance processes associated with these mechanisms and will be studied to determine the presence of water in the regolith.

\section{Mass and Volume Budgets}

The overall mass and volume budget of the lander is shown in Table 2. The budgets include a $15 \%$ contingency included for each subsystem. The overall margin for the concept stands at $9 \%$ mass and $34 \%$ volume margin. The propulsion and the landing system take up the bulk of the mass which will be optimized in the future.

Table 2. Mass and Volume budget of Arne-II lander.

\begin{tabular}{lcc}
\hline \hline \multicolumn{1}{c}{ Subsystem } & Mass (kg) & Volume (L) \\
\hline Avionics & 0.13 & 1.1 \\
Power & 1.1 & 0.8 \\
ADCS & 0.7 & 0.5 \\
Comms & 0.2 & 0.3 \\
Propulsion & 40.2 & 15 \\
Structure & 2.1 & 0.4 \\
Instruments & 0.4 & 0.6 \\
SphereX & 4.4 & 0.6 \\
Total & 49.2 & 27.8 \\
Margin & 4.8 & 15 \\
\hline \hline
\end{tabular}

\section{OPERATIONS}


After the lander lands near Mare Tranquilitatis pit, each of the three SphereX robots will be deployed using a spring-based deployment system. Each SphereX robots then needs to approach the pit entrance, enter the pit, and then explore the interiors of the pit by performing mapping and navigation. In this section we provide details of the mobility modes of SphereX and mapping and navigation techniques used to explore the pit.

\section{Mobility}

Mobility of SphereX is achieved through ballistic hopping with the help of a miniaturized propulsion system and 3-axis reaction wheel system. Two modes of ballistic hopping are identified for the robot to be able to explore Mare Tranquilitatis pit: a) Hard-landing mode for exploring short distances, and b) Soft-landing mode for pit entrance [28]. The hard-landing mode consists of three phases: i) Attitude correction phase, ii) Boost phase, and iii) Ballistic trajectory phase. During the attitude correction phase, the robot is oriented towards its desired attitude, and during the boost phase, the propulsion system provides a constant thrust $\|T\|$ for an optimal burn time $t_{b}$ while the attitude control system maintaining the desired attitude states. Finally, during the ballistic trajectory phase, the robot follows its natural trajectory due to the applied thrust and gravity. Similarly, the soft-landing mode, consists of four phases: i) Attitude correction phase, ii) Boost phase, iii) Ballistic trajectory + Attitude correction phase, and iv) Soft-landing phase. The first three phases are similar to the ones discussed above, but during the soft-landing phase, the propulsion system provides a constant thrust $\|T\|$ for an optimal burn time $t_{l}$ while the attitude control system maintaining the desired attitude states which are dependent on the instantaneous velocity of the robot.

\section{Mapping and Localization}

For Mapping and Localization of SphereX while exploring a target pit/lava tube, we use an ICP-based Pose-Graph SLAM algorithm on scans generated by the on-board LIDAR sensor [24]. The algorithm exploits scan registration algorithm and graphical model optimization to build an Iterative Closest Point (ICP)-based localization system in an unknown environment for SphereX equipped with 3D-LIDAR sensors. It consists of two main layers: ICP layer and Graph layer. The ICP layer comprises of keyframes $K_{i}$, and their associated 3D point cloud scans generated by the LIDAR sensor. To each keyframe $K_{i}$ is associated a node $n_{i}$ in the graph layer. The robot's body fixed frame $\mathcal{B}$ is always expressed with respect to the closest keyframe in the current local map. As the robot starts moving, the first 3D point cloud scan acquired by the LIDAR sensor is associated with the first keyframe $K_{0}$. Simultaneously, the corresponding node $n_{0}$ is created in the graph layer, and a factor representing the initial robot pose with respect to the reference frame $\mathcal{R}$ is added to the pose graph. Nominally, every time a new scan is available at the current robot body frame $\mathcal{B}$, the ICP algorithm finds the transformation that aligns it with the current local map, while estimating the robot pose in the process. A new keyframe is created if the overlap between the current scan and the current local map is lower than a threshold, otherwise the scan is just discarded. Next, while moving through the environment, if the robot detects a potential loop closing between two keyframes, the pose-graph optimization sequence is initiated. This 
is done by first building a local map around the oldest keyframe between the loop closing keyframes. Next, the ICP algorithm is called that tries to align the scan of the other loop closing keyframes with this local map. If the ICP is successful, a new factor is added to the graph between the variables associated with the loop closing keyframes, closing a loop in the graph level. Next an optimization sequence is triggered that uses the graph data. By using the optimization result, the keyframes are repositioned and local maps are reconstructed using these new repositioned keyframe values. The robot body fixed frame $\mathcal{B}$ being expressed in the closest keyframe, is also updated by the loop closing process. With multiple robots deployed inside the cave, a framework is developed to maintain a master map of the cave by merging maps generated by each robot [25]. Each robot $R^{i}$ generates its local map $m_{i}$ and pose estimates $p_{i}$ from its 3D Lidar scans. The local maps and pose estimates generated by each robot are then sent to a master robot, that generates the master map $M_{t}$ by performing pose-graph optimization. Once the pose-graph is optimized by the master, the trajectory updates $u_{i}$ are transferred back to each robot which are then used to correct their local maps and pose estimates.

\section{PLANS BEYOND}

Once the lava tubes are explored and evaluated for material composition, structural stability and detailed maps generated, they may form the basis for ideal habitats on the Moon, offering instant protection to astronauts from temperature variations, radiation and meteorite impacts. If they can be pressurized, they might be rendered suitable for habitation almost immediately. Otherwise, smaller habitats can be built inside their safe havens. The next section provides a conceptual design of a lunar base inside a lava tube with a pit entrance providing ways for entry and exit.

\section{Habitat Design}

The conceptual lunar base consists of a surface entity that expands into an underground lava tube extension through a pit as shown in Fig. 11. The underground base consists of multiple modules for living space, control and communication, power and electrical, food and bio systems, life support systems, storage facilities, etc. Multiple elevators are present around the pit entrance for transport of astronauts and goods from the underground base to the surface entity and vice versa. On the surface, the base consists of a power generation unit, antennas for communication and additional storage units for surface robots and machineries. Moreover, the entry and exit to and from the surface entity is done through multiple stiff airlock systems. The construction of the lunar base starts with the construction of tunnel modules and inflatable modules at first. The inflatable modules are attached in between two rigid structures to provide flexibility to the structure such that it can accommodate for change in size and shape of the rigid structure. The rigid structure provide support for the tunnel modules. For safe entry and exit into the tunnel modules on the surface a stiff airlock system is designed that can withstand drastic changes in air pressure. Air will be pumped into or out of the airlock to accommodate the crew's bodies to sudden change in pressure. Moreover, in order to connect two tunnel modules, a docking port is designed similar to the ones used in the International Space Station. The docking ports are attached to the end of each tunnel 
module that consists of a female and a male end. Hydraulic pistons on the male end push metallic cylinders into the corresponding socket in the female end. In this way, the tunnel modules can be expanded to construct the entire base.

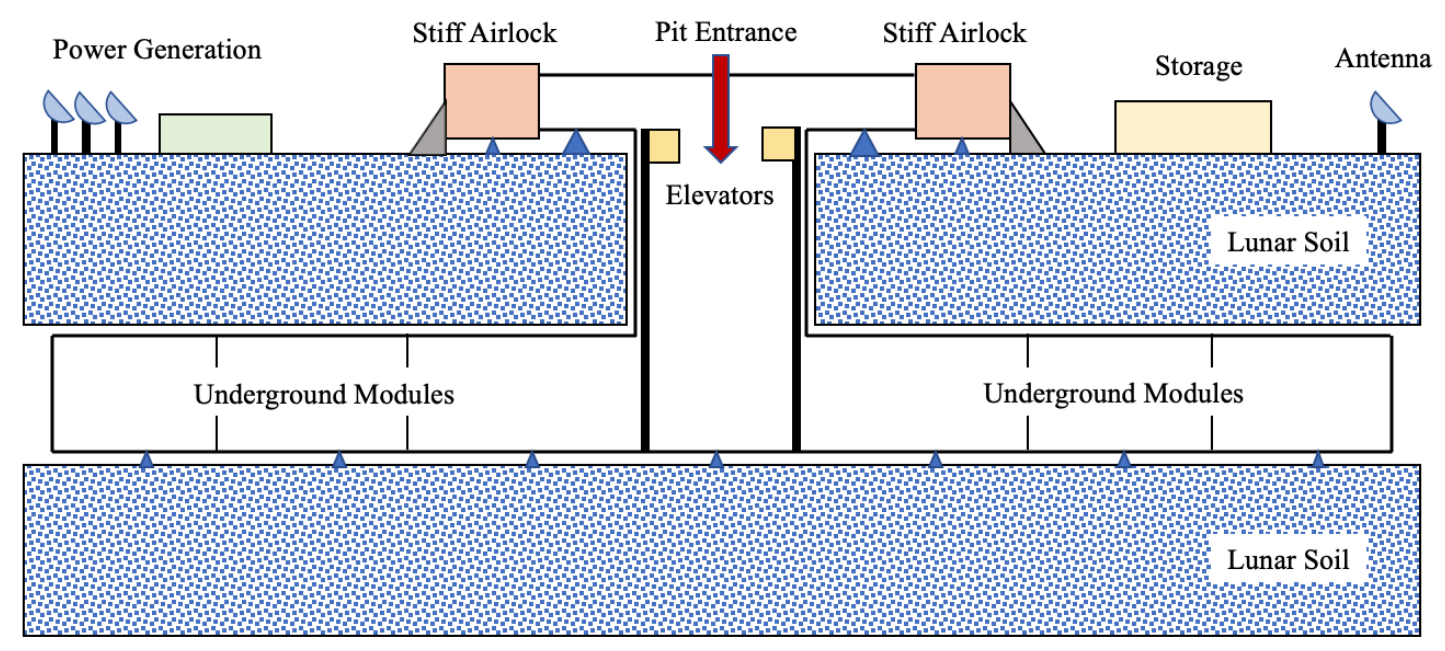

Fig. 11. Conceptual lunar base inside lava tubes.

The location of the base inside a lunar lava tube simplifies the design of the thermal system of the habitat, as the expected temperature inside the lava tubes will remain relatively constant at around $-25^{\circ} \mathrm{C}$. Additionally, using samples collected by the Apollo 11 mission and analyzed by Horai et al. [26], it is known that these lava flow tubes are made of non-porous basalt with high titanium content which have a relatively low thermal conductivity $\mathrm{k}$, and thus will be an effective natural insulator. The basic concept of the base's thermal insulation and wall structure loosely comes from the design of a spacesuit. This concept allows for the walls to be both ultra-durable and flexible in order to conform to and bend with the lunar lava flow tube walls. The base's walls will be composed of 6 different materials in 7 different layers. The inner and outer most layers will be a Nomex, Teflon, Kevlar ripstop woven fabric called OrthoFabric. This layer is abrasion, puncture, and tear resistant and acts as an insulator to protect the base from any unexpected extreme fluctuations in basalt temperature. The second most outer layer is a composite of aluminized Mylar, a Polyethylene Terephthalate film with evaporated aluminum on one or both faces. This layer serves as an excellent insulator and decreases thermal radiation, deflecting heat back into the base. The third layer from outside, is a nylon ripstop layer. This layer will serve as the pressure garment's last defense against any possible punctures from the outside. Next, the pressure garment layer, a noble-gas infused neoprene closed-cell foam is used to keep the base pressurized. The noble-gas infused closed-cell structure can reduce thermal conductivity of the neoprene by approximately $40 \%$ [27]. The second most inner layer is a heating bladder that will consist of a rubber sleeve which will be filled with water and heating elements. This layer will provide the heating and thermal regulation needed for the base in both the crew zones and the botany bay. Lastly, the final and inner most layer will be another layer of ortho-fabric that will act as a shield to the bladder layer as well as function as a thermal regulator, making sure the walls of the base are not too hot while letting heat through to warm the base. The total thickness of this multilayered multi fabric wall will be 20 millimeters. 
For food and bio-system the goal is to create sustainable food production while simultaneously reducing the reliance on $\mathrm{CO}_{2}$ scrubbers. The first food to be produced would be algae such as Arthrospira platensis, A. fusiformis, and A. maxima due to its simple needs, $\mathrm{CO}_{2}$ consumption, and $\mathrm{O}_{2}$ production. While growing the algae, the crew will be expected to assemble the necessary equipment for aeroponics so that lettuce, soybeans, potatoes, and strawberries can be grown. Since these plants produce a fruit or other edible offspring, they have increased $\mathrm{CO}_{2}$ consumption rates. Once a sustainable source of food is established, fish can be cultivated to provide some variety in the crew's diets. The fish can provide the crew with beneficial proteins and fats. They would also be capable of eating excess algae and create a way to reduce the uric acid buildup in the crew members from algae consumption. Fish eggs can be transported to the base to allow them to develop and act as a food source. Several potential species of fish have been selected such as common pleco, pond loach, and otocinclus catfish. The life support processes that are necessary include providing air that is consumable as well as clean drinking water. Oxygen production will largely come from lunar regolith, where it will be extracted using a calcium reduction process [28]. Carbon dioxide removal can be aided by the plants growing, but that will only account for about $25 \%$ of the minimum. The main Carbon dioxide removal device will be the same CDRA used on the ISS working in line with two carbon dioxide removal beds [29]. Water for the crew members will be produced using a hydrogen-oxygen combustion reaction as well as the mining of prospective ice inside lava tubes and craters. The water will be reused using a larger scale version of the water recycling system aboard the ISS, which features a urine processor assembly and a water processor assembly.

For a sustainable lunar base, in-situ resource utilization (ISRU) is one of the most important requirements. Although the primitive base can be built by transporting materials from Earth much like the International Space Station (ISS) but for maintaining the base for long term use and expanding it will require use of materials available on the surface of the Moon. In order to better actualize it, a network of automated robotic systems that can perform exploration and resource prospecting on the lunar surface and also inside the lava tubes is arguably the best option. In order to start this extensive but potentially high reward process, in situ sample collection and testing is the primitive option that would give us in depth insight into potential ore resources on the Moon. The communication system of the lunar base needs to meet two primary objectives: a) high speed communication between the lunar base and Earth, and b) reliable wi-fi connection inside the base and on the surface for crew members and exploration/mining robots. For transmitting information from the lunar base to Earth and vice-versa, laser communication has a significant advantage over traditional radio frequency (RF) communication. The Lunar Atmospheric Dust Environment Explorer (LADEE) mission already demonstrated two-way laser communication with a data rate of 622 megabits per second (Mbps) from lunar orbit to Earth [30]. The wi-fi network can be developed using RF communication by placing multiple routers strategically inside the base and also on the lunar surface. With multiple exploration rover deployed across the surface and deep inside the lava tube, 
the wi-fi network can be expanded over time. The goal of the power system is to generate and deliver sufficient power to the lunar base as it transitions from the initial surface base to the underground lava tube base. The primary challenge it experiences is the cycle of lunar day and night. The Moon takes 27.3 days to orbit around Earth, and the lunar day and night are approximately 2 weeks of continuous sunlight followed by darkness respectively. The lack of sunlight would require a battery system for power storage to run the base during the lunar nights. Solar collectors have an advantage over photo voltaic (PV) solar panels as solar collectors can produce energy from molten salt even during the lunar night while PV cells immediately stops producing power in the absence of sunlight. Solar collectors capture thermal energy from sunlight to heat up oil which heats up molten salt that drives a turbine. The electricity produced by the turbine will then be transmitted into the lava tube base through a transformer. For nominal operation of the base, the power system will be designed to produce $200 \mathrm{~kW}$ of power during the lunar day. Approximately $70 \%$ of the power will be used for operation of the base, and the excess $30 \%$ will be stored for use during the lunar night. A set of lithium ion batteries inside the underground base will be used to store power under optimal temperature and humidity conditions.

\section{CONCLUSION}

Presentation of this mission concept show the need for advancement in several areas of GNC to enable science exploration. The need for visual navigation to perform pinpoint landing with accuracy of $2 \mathrm{~km}$ to 500 meters is an important challenge. The potential opportunities will allow for routine but short missions to the surface of the Moon to obtain samples, perform in-situ analysis and setup instruments. The technology to perform these feats are already there from previous lunar surface missions. The technology does not require the spacecraft be entirely autonomous. With the gateway being nearby, it is possible to partially teleoperate these crafts to minimize on mission operational complexity. Nevertheless, it is the miniaturization of this technology and use of COTS parts that will be new. This includes advancement in smart-navigation cameras, altitude sensing radars, miniature LIDAR and flash-cameras to name a few. Such technology will be critical to perform the required adjustments during soft-landing on the lunar surface. Apart from the advancement in smart sensing, we have introduced landers that can deploy from the 27U CubeSat form factor. This is another critical technology advancement. The lander needs to withstand hard landings, not tip over and be upright for positioning communications antennas and navigation cameras.

Technologies we have omitted to keep the mission simple includes the required thermal and power technologies to keep a small lander alive during the lunar night. This maybe a requirement for missions that need to deploy permanent surface instruments. In other cases, the 6-12 days of mission time on the lunar surface appears enough to obtain the required science data. Overall the proposed Lunar Gateway offers a credible steppingstone to perform exploration of the Moon. CubeSats while still being at their infancy could be used to routinely explore and access all parts of the Moon thus avoiding the risks and high costs associated with directly sending astronauts. Just as 
the ISS and its vicinity has become a proving ground in Low Earth Orbit (LEO), the setup also provides an ideal proving ground to advance deep-space technology, terrain navigation technology and technology to perform pin-point landing on the Moon. All of these technologies need to advance for us to realize permanent bases on the Moon, Mars and the asteroids.

Constructing a base inside a lava tube simplifies the design, as they are sheltered from hazards such as temperature fluctuation, radiation, and meteorite impacts. We provided a feasibility study of the major subsystems of such a base namely structural design, thermal design, food and bio-systems, life support system, robotic system, communication system, and power system. These conceptual feasibility studies identified the major technologies required for setting up the base and provided a path forward towards development of some of the lunar lava tubes into critical habitable structures in support of permanent human presence on the Moon.

\section{REFERENCES}

[1] National Academies of Sciences, Engineering, and Medicine. 2011. Vision and Voyages for Planetary Science in the Decade 2013-2022. Washington, DC: The National Academies Press. doi: 10.17226/13117.

[2] R.V. Wagner and M.S. Robinson, "Distribution, formation mechanisms, and significance of lunar pits," Icarus, Vol. 237, pp. 52-60, 2014.

[3] M.S. Robinson et al., "Lunar reconnaissance orbiter camera (LROC) instrument overview," Space Science Reviews, Jan. 2010, Vol. 150, No 1-4, pp.81-124.

[4] G. Heiken, D. Vaniman and B. French, "Lunar Sourcebook: A User's Guide to the Moon," Cambridge Univ. Press, 1991.

[5] National Academies of Sciences, Engineering, and Medicine. 2016. NASA Space Technology Roadmaps and Priorities Revisited. Washington, DC: The National Academies Press. doi: $10.17226 / 23582$.

[6] National Academies of Sciences, Engineering, and Medicine. 2016. Achieving Science with CubeSats: Thinking Inside the Box. Washington, DC: The National Academies Press.

[7] R. Zubrin, "Moon Direct: A Cost-Effective Plan to Enable Lunar Exploration and Development," AIAA SciTech 2019 Forum, 2019, San Diego, CA.

[8] J. Schoolcraft, A. Klesh, and T. Werne, "MarCO: Interplanetary Mission Development On a CubeSat Scale,” AIAA SpaceOps 2016 Conference, 2016, 2491.

[9] R. Staehle, wt. al., "Interplanetary CubeSats: Opening the Solar System to a Broad Community at Lower Cost," Journal of Small Satellites, 2013, Vol 2, No. 1, pp. 161-166.

[10] A. Babuscia, T. Choi, KM Cheung, J. Thangavelautham, M. Ravichandran, A. Chandra "Inflatable antenna for CubeSat: Extension of the previously developed S-Band design to the X-Band," AIAA Space 2015 Conference, 4654.

[11]R. Pothamsetti and J. Thangavelautham, "Photovoltaic electrolysis propulsion system for interplanetary CubeSats," 2016 IEEE Aerospace Conference, Big Sky, MT, 2016, pp. 1-10.

[12] J. Thangavelautham, M. S. Robinson, A. Taits, et al., "Flying, hopping Pit-Bots for cave and lava tube exploration on the Moon and Mars" 2nd International Workshop on Instrumentation for Planetary Missions, NASA Goddard, 2014.

[13] H. Kalita, A. Ravindran, S. Morad, J. Thangavelautham, "Path Planning and Navigation Inside OffWorld Lava Tubes and Caves," IEEE/ION PLANS Conference, 2018.

[14]H. Kalita, J. Thangavelautham, “Automated Multidisciplinary Design and Control of Hopping Robots for Exploration of Extreme Environments on the Moon and Mars," 70th International Astronautical Congress (IAC), Washington D.C., USA, 2019, 21-25 October. 
[15]H. Kalita, A. S. Gholap, J. Thangavelautham, "Dynamics and Control of a Hopping Robot for Extreme Environment Exploration on the Moon and Mars," IEEE Aerospace Conference, Big Sky, USA, 2020, 7-14 March.

[16] J. Haruyama, et. al., (2009) "Possible lunar lava tube skylight observed by SELENE cameras," Geophysics Research Letters 36, L21206.

[17] M.S. Robinson, J. Thangavelautham, R. Wagner, V. Hernandez, J. Finch, "Arne - Exploring the Mare Tranquilitatis Pit," American Geophysical Union Fall Meeting, 2014.

[18] H. Kalita, et. al., "GNC Challenges and Opportunities of CubeSat Science Missions Deployed from the Lunar Gateway," Advances in the Astronautical Science, 2019.

[19] Yu, M., Cui, H., Tian, Y., "A new approach based on crater detection and matching for visual navigation in planetary landing," Advances in Space Research 53 (2014) 1810-1821.

[20] Glavin, D. P., et. al., "Volatile Analysis by Pyrolysis of Regolith for Planetary Resource Exploration," IEEE Aerospace Conference, 2012.

[21] Getty, S. A., et. al., "Development of an evolved gas-time-of-flight mass spectrometer for the Volatile Analysis by Pyrolysis of Regolith (VAPoR) instrument," International Journal of Mass Spectrometry 295 (2010) 124-132.

[22] Roman, P. A., et al, "A Miniature MEMS and NEMS enabled Time-of-Flight Mass Spectrometer for Investigations in Planetary Science," Proceedings of SPIE - The International Society for Optical Engineering 6959, 2008.

[23] Seshadri, S., et al., "Using Electrical Impedance Spectroscopy to Detect Water in Planetary Regoliths," Astrobiology, vol. 8, no. 4, 2008.

[24]E. Mendes, P. Koch, S. Lacroix, "ICP-Based Pose-Graph SLAM," International Symposium on Safety, Security and Rescue Robotics, pp. 195-200, 2016.

[25]R. Dube, et al., "An Online Multi-Robot SLAM System for 3D LiDARs," IEEE/RSJ International Conference on Intelligent Robots and Systems (IROS), 2017.

[26] Horai, K., and Fujii, N. (1972). "Thermophysical properties of lunar material returned by Apollo missions." The Moon, 4(3-4), 447-475. doi:10.1007/bf00562011

[27] Moran, J., et al. (2018). "Noble-gas-infused neoprene closed-cell foams achieving ultra-low thermal conductivity fabrics." RSC Advances, 8(38), 21389-21398. doi:10.1039/c8ra04037k

[28]Landis, G. A. (2011). "Calcium Reduction as a Process for Oxygen Production from Lunar Regolith." 49th AIAA Aerospace Sciences Conference, Orlando, FL.

[29] Sherif, D., and Knox, J. (2005) "International Space Station Carbon Dioxide Removal Assembly (ISS CDRA) Concepts and Advancements." SAE Technical Paper, 2005-01-2892, doi:10.4271/2005-01-2892.

[30] Boroson, D. M., et al. (2009) "The Lunar Laser Communications Demonstration (LLCD)." 3rd IEEE International Conference on Space Mission Challenges for Information Technology. 